\title{
BER Performance of Space-Time Coded MMSE DFE for Wideband Code Division Multiple Access (WCDMA)
}

\author{
Sanjay Kumar SHARMA ${ }^{1}$, S. Naseem AHMAD ${ }^{2}$ \\ ${ }^{1}$ Dept. of Electronics and Communication Engg., Krishna Institute of Engg. and Technology, Ghaziabad, India \\ ${ }^{2}$ Dept. of Electronics and Communication Engg, Jamia Millia Islamia, Delhi, India \\ Email: sanjaysharma1515@yahoo.co.in \\ Received January 20, 2009; revised March 1, 2009; accepted April 27, 2009
}

\begin{abstract}
In recent times, there has been growing interests in integration of voice, data and video traffic in wireless communication networks. With these growing interests, WCDMA has immerged as an attractive access technique. The performance of WCDMA system is deteriorated in presence of multipath fading environment. The paper presents space-time coded minimum mean square error (MMSE) Decision Feedback Equalizer (DFE) for wideband code division multiple access (WCDMA) in a frequency selective channel. The filter coefficients in MMSE DFE are optimized to suppress noise, intersymbol interference (ISI), and multiple access interference (MAI) with reasonable system complexity. For the above structure, we have presented the estimation of BER for a MMSE DFE using computer simulation experiments. The simulation includes the effects of additive white Gaussian noise, multipath fading and multiple access interference (MAI). Furthermore, the performance is compared with standard linear equalizer (LE) and RAKE receiver. Numerical and simulation results show that the MMSE DFE exhibits significant performance improvement over the standard linear equalizer (LE) and RAKE receiver.
\end{abstract}

Keywords: Decision Feedback Equalizer, Multiple Access Interference (MAI), RAKE Receiver, Transmit Diversity, Wideband Code-Division Multiple-Access (WCDMA), Bit Error Rate (BER)

\section{Introduction}

During the period of last one decade, the large demands for wireless services and high data speeds have driven the wireless cellular networks to a tremendous growth. These large demands require some advanced techniques like WCDMA that can support more users and higher data rates. WCDMA has been accepted as standard access method for the third and fourth generation wireless systems. The WCDMA system assigns each user a specific signature sequence from the signature set. One limiting factor in the capacity (i.e. the number of users) of the WCDMA systems is the multiple access interference (MAI). In fact, in WCDMA system, multiple access interference (MAI) and intersymbol interference (ISI) are caused by multipath dispersion and are major problems. These problems cannot be efficiently suppressed by conventional RAKE receivers. The MAI caused by one user is usually small, but as the number of interferers or their power increases, effect of MAI becomes noticeable. To alleviate the effect of MAI, a number of multiple user detection methods have been proposed in literature in recent years [1]. Usually, the multiuser receiver can perform much better than the conventional correlator based receiver, but at the cost of increased system complexity. For uplink channel, the increased complexity is not a big issue since the base station may be equipped with some powerful computing processors. Whereas, a mobile terminal is limited by cost and size. Therefore, it will be very difficult to use the multiuser receiver for the downlink channel. But, irrespective of this problem, there is a strong emphasis to improve the performance of the downlink channel in WCDMA.

Another limiting factor in the cellular systems is fading. An usual technique for combating fading is spatial 
diversity. In WCDMA systems, two methods of spatial diversity and interference cancellation can be combined to increase the system performance and capacity. The combination of MUD and receive diversity techniques has been proposed in [2,3]. In third generation WCDMA systems, the processing transmit gain may be very small. This makes the use of diversity quite effective. Transmit diversity can be used to alleviate fading efficiently. There are several forms of transmit diversity. In openloop scenarios, where the transmitter does not have the channel state information, space-time transmit diversity (STTD) is generally used. When channel state information is available, closed-loop transmit diversity such as beam forming can be used. Over the period of last decade, various transmit diversity schemes have been proposed in modern wireless communications to combat fading. Among various proposed techniques, Alamouti's space-time block code [4], called as space- time transmit diversity (STTD), is one of the most effective solutions for two transmit antennas. Because of its effectiveness, Alamouti's space-time block code has been adopted for third generation WCDMA systems in indoor applications for high data rate.

WCDMA downlink has two interesting features. One is that all transmissions are synchronized and the other is that the spreading codes can be orthogonal. By taking advantages of these features, the chip-level equalization has been proposed to mitigate MAI with a despreader [5, 6]. A despreader can mitigate the MAI after chip-level equalization to restore the orthogonality. In [7], it has been shown that a receiver with a chip-level equalizer can be easily implemented with adaptive algorithms. Unfortunately, the performance of the receiver with a chip-level linear equalizer (LE) is not significantly better than the rake receiver, unless the receiver of the mobile station is equipped with multiple receiving antennas or uses over sampling. Because the LE cannot perfectly suppress the miltiptah interference with a single receive antenna and chip rate sampling, a residual multipath interference exists. Thus, the MAI cannot be completely removed by the correlator or despreder. To avoid this, multiple receive antennas or a higher sampling rate can be used for the chip level minimum mean square error linear equalizer (MMSE-LE) [10]. Decision feedback equalization is a powerful equalization technique that provides postcursor ISI cancellation with reduced noise enhancement and is widely used to offer better steadystate performance than a linear equalizer (LE). Recent research has been devoted to the receiver design using zero forcing [11], minimum MSE (MMSE) [12] methods.

The zero forcing receivers can completely suppress the ISI and multi-user interference under certain conditions. However, explicit knowledge of all the signature wave- forms is required and the noise may be enhanced. Hence, the receiver designed by using the MMSE criterion seems to be better than zero-forcing receivers in terms of their bit error rate (BER) performance. In this paper, we investigate and analyze a minimum mean-square error (MMSE) decision feedback equalizer (DFE) for spacetime coded WCDMA downlink channel to achieve better performance than the chip level LE and a RAKE receiver in a frequency selective channel.

The rest of the paper is organized as follows. The signals and system models have been introduced in section 2.In subsection 2.1 we have discussed the basic spacetime encoder in WCDMA. In subsection 2.2,we have presented the structure of a traditional decision feedback equalizer (DFE). The space-time coded decision feedback equalizer (DFE) has been formulated in subsection 2.3. Subsection 2.4 presents the mathematical analysis. In section 3 , we have formulated the simulation environments. Computer simulation results are presented in section 4 to see the performance and we conclude the paper with some remarks in section 5 .

\section{Signal and System Models}

\subsection{Basic Space-Time Encoder in WCDMA}

Figure 1 shows the basic space-time transmit diversity (STTD) encoder in WCDMA. We consider two symbols periods, 0 and 1 , over which two symbols are sent. During symbol period 0 , symbol $c_{0}$ is sent on transmit antenna $A$ and $c_{1}{ }^{*}$ is sent on antenna $B$.

During symbol period $1, c_{1}$ is sent on transmit antenna $A$ and $c_{0}{ }^{*}$ is sent on antenna $B$. It is assumed that the same channelization code is used to send these STTD encoded symbols. But, the pseudo-random scrambling codes are different for different symbol periods. Let $h_{j i}(t)$ denote the continuous-time impulse response of the multipath channel from transmitter antenna $i$ to the receive antenna $j$. A time-variant multipath signal propagation through the mobile cellular radio channel can be modeled as:

$$
h_{j i}(t)=\sum_{q=0}^{Q-1} \alpha_{j i, q}(t) e^{j \theta_{j i, q}(t)} \delta\left(t-\tau_{j i, q}(t)\right)
$$

where $Q$ is the number of channel multipath, $\delta(\cdot)$ is

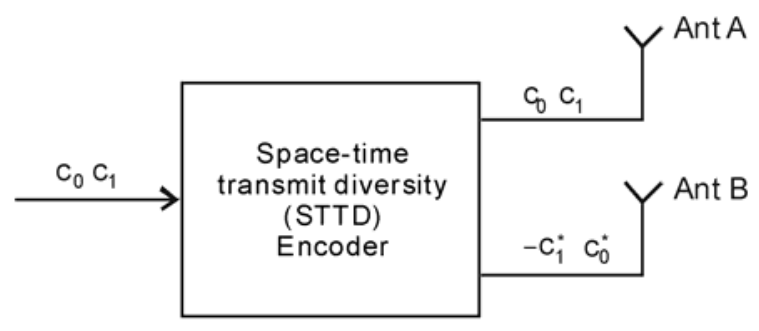

Figure 1. Space-time encoder in WCDMA. 
the Dirac-delta function, and $\quad j i, q(t), \quad j i, q(t)$ and $\quad{ }_{j i, q}(t)$ are the time-variant attenuation, phase distortion and propagation delay of the qth path from transmit antenna $i$ to receive antenna $j$, respectively.

\subsection{Traditional Decision Feedback Equalizer}

We first describe a traditional decision feedback equalizer (DFE) receiver, upon which a two dimensional DFE for WCDMA system builds. Figure 2 shows a discretetime complex base band model for the conventional DFE. The DFE consists of a feed forward filter $f(n)$ and a feedback filter $b(n)$, where $n$ is the symbol index. Since the feedback filter sits in a feedback loop, it must be strictly causal. The signal propagates through a discrete time-variant frequency selective fading channel $h(n)$.

Also, r(n) $\rightarrow$ received signal, d(n) $\rightarrow$ transmitted symbols information, $\hat{d}(n) \rightarrow$ output of DFE.

\subsection{Space-Time Coded Decision Feedback Equalizer (DFE)}

We concentrate on WCDMA downlink channel with transmit diversity. The system employs two transmit antennas at the transmitter side and one receive antenna at the receiver side. We assume that there are $\mathrm{K}$ active users in the cell under consideration and that the intercell interference is negligibly small in cellular scenario. Also, there are $\mathrm{M}$ transmit antennas and $\mathrm{V}$ receive antennas in the system.

Now, the transmitted signal of user $\mathrm{k}$ from antenna $\mathrm{m}$, represented by $x_{k}^{(m)}(t)$, is given by

$$
x_{k}^{(m)}(t)=\sum_{n=0}^{N-1} A_{k} c_{k}^{(m)}(n) w_{k, n}\left(t-n T_{s}\right)
$$

where, $c_{k}^{(m)}(n), n=0, \ldots, N-1$, is the $k$ th user's spacetime coded data sequence to be transmitted from antenna $m$ within a specific time-frame

$A_{k}$ is the average amplitude of the $k$ th user

$T_{s}$ is the symbol duration

$w_{k, n}(t)$ is the signature waveform of user $k$ at the nth symbol period

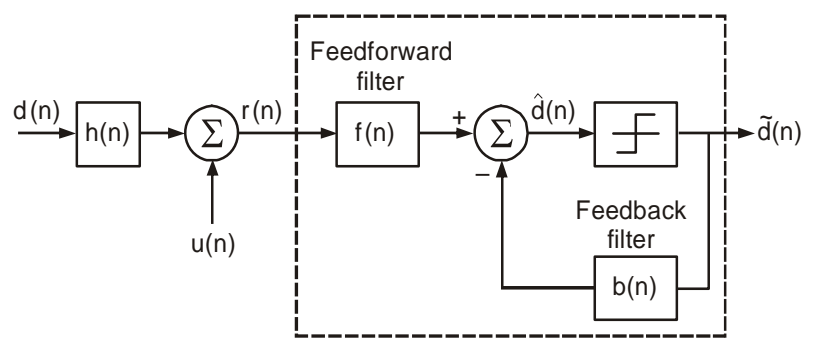

Figure 2. The traditional DFE structure.
The signature waveform $w_{k, n}(t)$ is represented as

$$
w_{k, n}(t)=\frac{1}{\sqrt{G}} \sum_{i=0}^{G-1} s_{k, n}(i) p\left(t-i T_{c}\right)
$$

where $G$ is the processing gain,

$T_{c}$ is the chip duration,

$p(t)$ is the chip pulse shape signal

$s_{k, n}(i)$ is the $k$ th user's signature sequence

The spread signal is transmitted over the frequency and time selective channels. The channels from the two base station antennas to the receiver are modeled as Rayleigh multipath fading channels. Then, according to basic equation (1), the impulse response of the channels between the base station transmitter and the mobile station receiver is

$$
h^{(m)}(t)=\sum_{q=0}^{Q-1} h_{q}^{(m)} \delta\left(t-t_{q}\right), \quad m=1,2
$$

where $Q$ is the number of paths,

$h_{q}(m)$ is a complex coefficient which is used to model the qth path

$t_{q}$ is the delay related to the $q^{\text {th }}$ path.

We assume that the number of paths and their delay times are equal for the two channels. Finally, the received signal from all $K$ users at a mobile base station after demodulation is given by

$$
r(t)=\sum_{m=1}^{2} \sum_{q=0}^{Q-1} \sum_{k=0}^{K-1} h_{q}^{(m)} x_{k}^{(m)}\left(t-t_{q}\right)+u(t)
$$

where $u(t)$ is the additive white Gaussian noise (AWGN) with noise variance of $\sigma^{2}$.

It may be more convenient to consider a discrete-time signal model. Accordingly, the received signal sequence is written as

$$
r(n)=\sum_{m=1}^{2} \sum_{q=0}^{Q-1} \sum_{k=0}^{K-1} h_{q}^{(m)} x_{k-q}^{(m)}+u_{k}
$$

where $r(n)$ is the received signal sequence, and $u_{k}$ is the white noise sequence.

\subsection{Mathematical Analysis}

To achieve transmit diversity; we use Alamouti's spacetime block code for two transmit and one receive antennas [4]. In a space-time block-level encoding scheme, each block of $2 \mathrm{~N}$ information symbols $d_{k}(n), n=0, \ldots$, $2 \mathrm{~N}-1$, is split into two blocks of odd and even symbols, each having length $N$. Further, these two blocks are space-time encoded and transmitted during two subsequent periods, each having a duration of $N T_{s}$. This means that for the first and second period, we have 


$$
\left.\begin{array}{rl}
c_{k}{ }^{(1)}(n) & =d_{k}(2 n) \\
c_{k}^{(2)}(n) & =d_{k}(2 n+1) \\
c_{k}{ }^{(1)}(n+N) & =-d_{k}{ }^{*}(2 n+1) \\
c_{k}{ }^{(2)}(n+N) & =d_{k}{ }^{*}(2 n)
\end{array}\right\}
$$

where $n=0, \ldots . ., N-1$.

If we place a guard-time $t_{g}$ between two transmission periods, the received signal $r(t)$ consist of two non- interfering signals. Now, let $d_{o}(2 n)$ and $d_{o}(2 n+1)$ be the desired symbols to be detected at the receiver. According to Figure 2, $r(t)$ is first allowed to pass through the two matched filters which are matched to the corresponding spreading waveforms of the user and then sampled at times $t_{v}, v=0, \ldots, i-1$. Then, we have

$$
\begin{gathered}
r_{1}=\int_{0}^{T_{s}} w_{0, n}^{*}(\tau) r\left(\tau+n T_{s}+t_{v}\right) d \tau \\
r_{2}=\left[\int_{0}^{T_{s}} w_{0, n+N}^{*}(\tau) r\left(\tau+(n+N) T_{s}+t_{v}+t_{g}\right) d \tau\right]^{*}
\end{gathered}
$$

For ideal correlation properties of signature waveforms, there shall be no ISI and MAI in above samples. Thus, in ideal scenario, a RAKE receiver and a linear equalizer (LE) can work as optimum receiver [8]. But, in practice, because of non-zero auto and cross-correlations of the shifted signature waveforms, ISI and MAI occur and hence the performance of a RAKE receiver deteriorates in highly interference environments. Thus, to achieve the performance improvement, we use a spacetime coded DFE structure with two transmit and one receive antenna as shown in Figure 3. Now, the input to the first decision part, to detect $d_{0}(2 n)$, is written as

$$
\begin{gathered}
d_{e}=\sum_{q=0}^{L_{f}-1}\left[f_{11}\left(L_{f}-1-q\right) r_{1, q}+f_{21}\left(L_{f}-1-q\right) r_{2, q}\right]+ \\
\sum_{m=1}^{L_{b}}\left[b_{11}\left(L_{b}-m\right) \hat{d}_{o}(2 n-2 m)+b_{21}\left(L_{b}-m\right) \hat{d}_{o}(2 n-2 m+1)\right]
\end{gathered}
$$

The expression in equation (10) may be written in matrix form as under:

$$
\mathbf{d}_{\mathbf{e}}=\mathbf{F}^{\mathrm{H}} \mathbf{Y}
$$

Here,

$$
\begin{gathered}
\mathbf{F}^{\mathbf{H}}=\left[f_{11}\left(L_{f}-1\right), \ldots, f_{11}(0), f_{21}\left(L_{f}-1\right), \ldots \ldots,\right. \\
\left.f_{21}(0), b_{11}\left(L_{b}-1\right), \ldots, b_{11}(0), b_{21}\left(L_{b}-1\right), \ldots ., b_{21}(0)\right]
\end{gathered}
$$

And

$$
\begin{aligned}
& \mathrm{Y}=\left[r_{1,0}, \ldots . ., r_{1}, L_{f}-1, r_{2,0}, \ldots . ., r_{2}, L_{f}-1, d_{o}(2 n-2)\right. \\
& \left.\ldots . ., d_{o}\left(2 n-2 L_{b}\right), d_{0}(2 n-1), \ldots . ., d_{o}\left(2 n-2 L_{b}+1\right)\right]^{\mathrm{J}}
\end{aligned}
$$

The mean square error (MSE) is given by

$$
\varepsilon=E\left\{\left|d-d_{0}(2 n)\right|^{2}\right\}
$$

Now, to achieve the performance improvement, we have to minimize the mean square error (MSE). For that purpose, we must decide appropriate value of weight vector. The solution to the MMSE problem is given by

$$
\mathbf{F}^{\mathbf{H}}=\mathbf{A}^{-\mathbf{1}} \mathbf{G}
$$

where

$$
\mathbf{A}=\text { E. }\left\{\mathbf{Y} \mathbf{Y}^{\mathrm{H}}\right\}
$$

and

$$
\mathbf{G}=\mathrm{E}\left\{\mathbf{Y} \mathbf{Y} \boldsymbol{d}_{o}^{*}(2 n)\right\}
$$

To determine matrix $\mathbf{A}$ and vector $\mathbf{G}$, we must know the channel model and also we assume that the interfering user's signature codes and all the information symbols are independent random sequences. Now, the minimum mean-square error for the system can be written as

$$
\boldsymbol{\varepsilon}_{\mathrm{min}}=1-\mathbf{G}^{\mathrm{H}} \mathbf{S}^{-1} \mathbf{G}
$$

We can estimate the overall signal-to-noise ratio per symbol, using the Gaussian approximation, from the MMSE as [9]

$$
\mathrm{SNR}=\left(1-\boldsymbol{\varepsilon}_{\min }\right) / \boldsymbol{\varepsilon}_{\min }
$$

Hence, the bit error rate (BER) can be approximated as under:

$$
\begin{gathered}
\mathrm{P}_{\mathrm{e}}=Q(\sqrt{S N R}) \\
\mathrm{P}_{\mathrm{e}}=Q\left(\sqrt{\left(1-\boldsymbol{G}^{H} \boldsymbol{A}^{-1} \boldsymbol{G}\right) / \boldsymbol{G}^{H} \boldsymbol{A}^{-1} \boldsymbol{G}}\right)
\end{gathered}
$$

From above expression, it is obvious that $P_{e}$ depends upon the coefficients of the channel and signature waveform of the desired user.

\section{Simulation Environments}

We study the performance of a chip-rate DFE in a WCDMA downlink channel using QPSK modulation scheme and a spreading factor of 32 . We have assumed Rayleigh fading channels and channel coefficients as complex Gaussian random variables. The system transmits the data at $480 \mathrm{kbps}$, and the frame structure of $10 \mathrm{~ms}$ duration includes 15 slots. Each slot consists of 160 QPSK symbols that are spread by the Walsh-Hadmard code with period 32. As a whole, a chip rate of 3.84 $\mathrm{Mc} / \mathrm{s}$ is used. The channel taps are each subject to the Rayleigh fading around their mean value. Throughout the simulation work, the estimation is performed at $\mathrm{S} / \mathrm{N}$ $=10 \mathrm{~dB}$. We assume 16 active users within the same cell/frequency. However, the actual number of users may be more depending upon the service. 


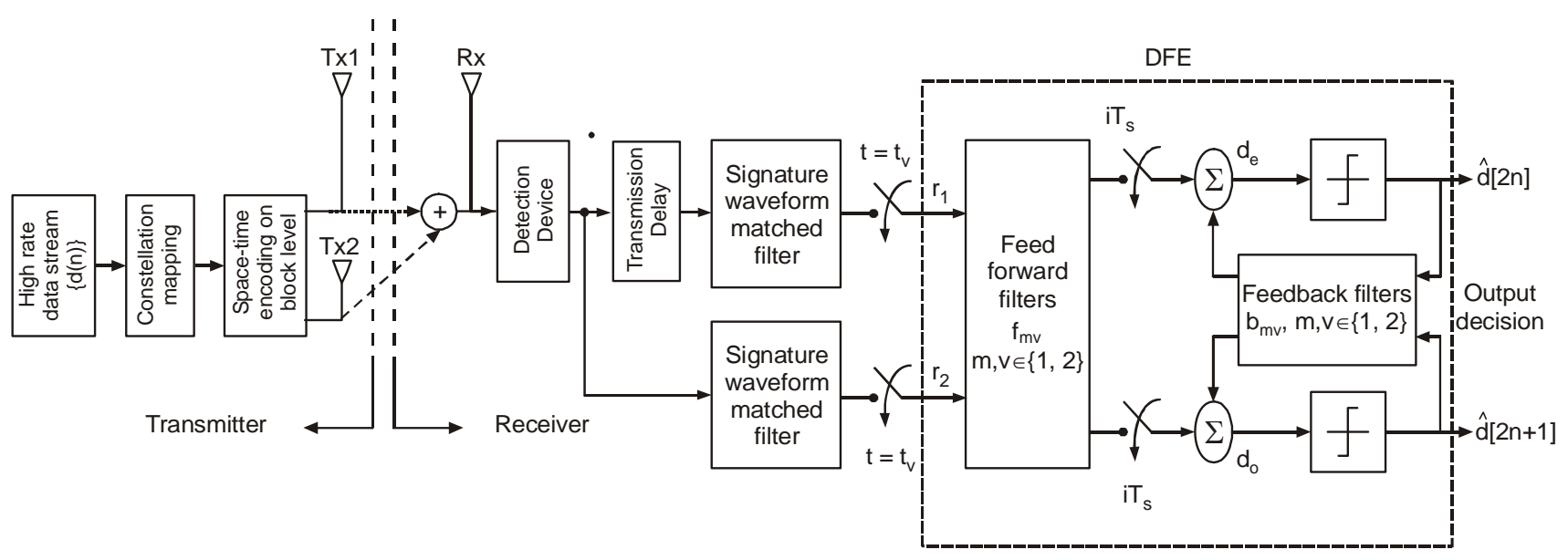

Figure 3. System model.

For two transmit antenna structure shown in figure 3, the feed forward filters are represented by $f_{m v}(n), m, v \in\{1$, 2 \}, each having $L_{f}$ taps and sampling is performed at $i$ times the chip rate. Also, the feedback filters are represented by $b_{m v}(n), m, v \in\{1,2\}$, each having $L_{b}$ taps and operating at the symbol rate. Throughout the simulation work, the Rayleigh fading channel uses $Q=3$ paths and the number of DFE feed-forward taps, $L_{f}$ is equal to four. Because of shorter length of channel memory than the period of symbols, it is evident that ISI is produced only by the adjacent symbols. Thus, the DFE feedback filters each requires only single tap. This means that we take $L_{b}$ $=1$. Further, simulation has been performed on over 4000 blocks each consisting of 800 space-time coded symbols and also channel is assumed constant during each frame.

Table 1. List of parameters for simulation.

\begin{tabular}{clc}
\hline S. No. & \multicolumn{1}{c}{ Simulation Parameter } & Value \\
\hline 1 & Carrier frequency & $2 \mathrm{GHz}$ \\
2 & Modulation technique & QPSK \\
3 & Data Rate & $480 \mathrm{kbps}$ \\
4 & Processing gain & 32 \\
5 & Signature or Spreading code & Walsh-Hadamard \\
6 & Number of multipaths & Code \\
7 & Number of feed forward taps & 4 \\
8 & Number of feedback filter taps & 1 \\
9 & Chip rate & $3.84 \mathrm{Mc} / \mathrm{s}$ \\
10 & No. of transmit antenna & 2 \\
11 & No. of receive antennas & 1 \\
12 & Performance parameter & BER \\
13 & Channel Model & Rayleigh multipath \\
\end{tabular}

\section{Simulation Results}

In Figure 4, the bit error rate (BER) has been plotted as a function of average signal to noise per bit $\left(E_{b} / N_{o}\right)$ for Rake receiver, adaptive linear equalizer (LE) and DFE with two transmit antennas (two dimensional DFE) and one receive antenna. In this case, we have assumed 3-paths channels and 16 active users within the cell of interest. Also, the number of feed-forward taps, $L_{f}$ for two-dimensional DFE has been taken equal to 4. Simulation has been performed on over 4000 blocks each consisting of 800 space-time coded symbols. From Figure 4 , it may be observed that as $E_{b} / N_{o}$ increases, BER of DFE fall faster than RAKE and linear equalizer. At higher values of $E_{b} / N_{o}$, the MAI dominates and the performance curve of RAKE receiver approaches a saturation level. At this point, significant performance improvement is achieved by DFE. In Figure 5, the bit error rate (BER) has been plotted as a function of number of active users for Rake receiver, adaptive linear equalizer (LE) and DFE with two transmit antennas (two dimensional DFE) and one receive antenna at $E_{b} / N_{o}=20 \mathrm{~dB}$. In this case also, we have assumed 3-paths channels and the number of feed-forward taps, $L_{f}$ for two-dimensional DFE has been taken equal to 5 . It can be observed as the number of users increases, MMSE DFE offers better performance than Rake receiver, adaptive linear equalizer (LE). Figure 6 reveals that as the speed of the mobile becomes higher, it is more difficult for the equalizer to manage successfully the variation of channel. It results in the increase of MAI in the receivers. Consequently, the performance of the DFE deteriorates as the speed of the mobile becomes higher. Also, the variation of the channel does not affect the performance of the rake receiver since the performance degradation is only due to the channel estimation error. 


\section{Conclusions}

In the paper, we have investigated the receiver using MMSE DFE for WCDMA downlink with space-time transmit diversity in a frequency selective channel. Bit error rate (BER) of various systems has been calculated using Gaussian approximation. In simulation curves, we have shown the BER performances with respect to the number of users, $E_{b} / N_{o}$ and mobile speed. It is observed that with the increase in number of transmissions, the performance of all the receivers, i.e., adaptive LE, the Rake and the DFE, becomes worse because of increase in intersymbol interference (ISI) and multiple access interference (MAI). According to simulation curves, a MMSE DFE outperforms an adaptive LE and a RAKE receiver for the large number of users and also when BER is compared with respect to the $E_{b} / N_{o}$ values. This happens because a MMSE DFE suppresses interference

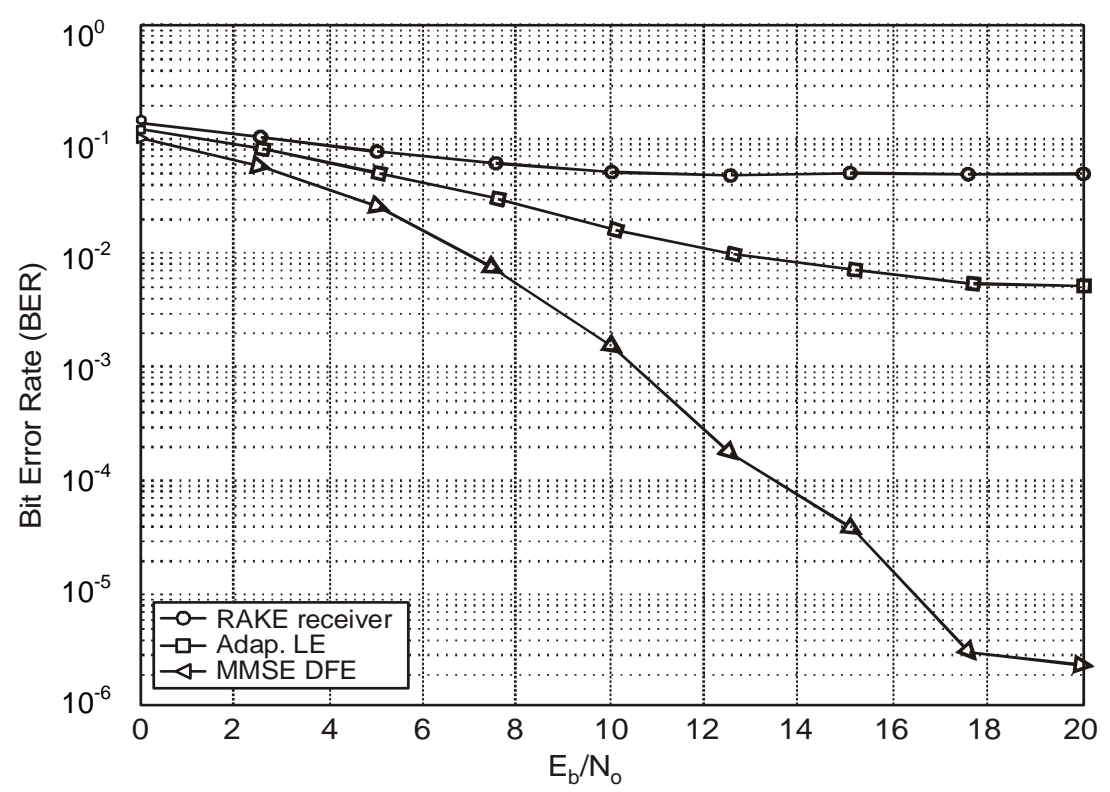

Figure 4. BER versus $E_{b} / N_{o}$.

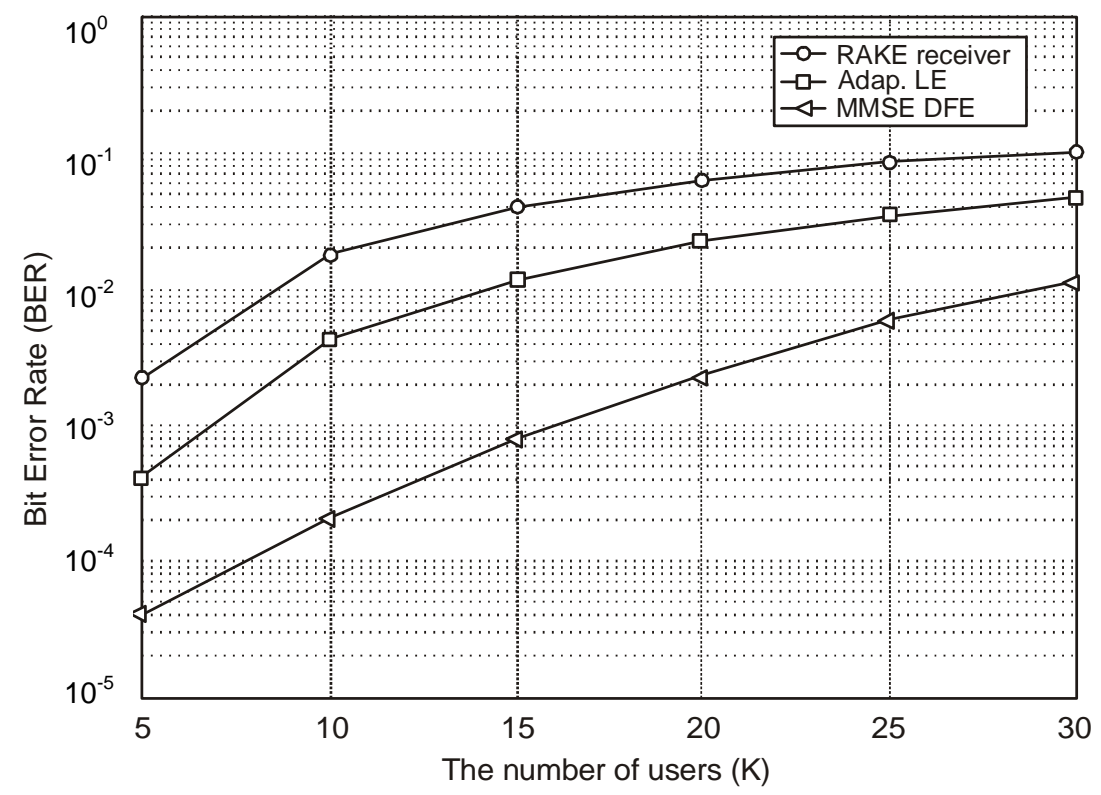

Figure 5. BER versus the number of active users. 


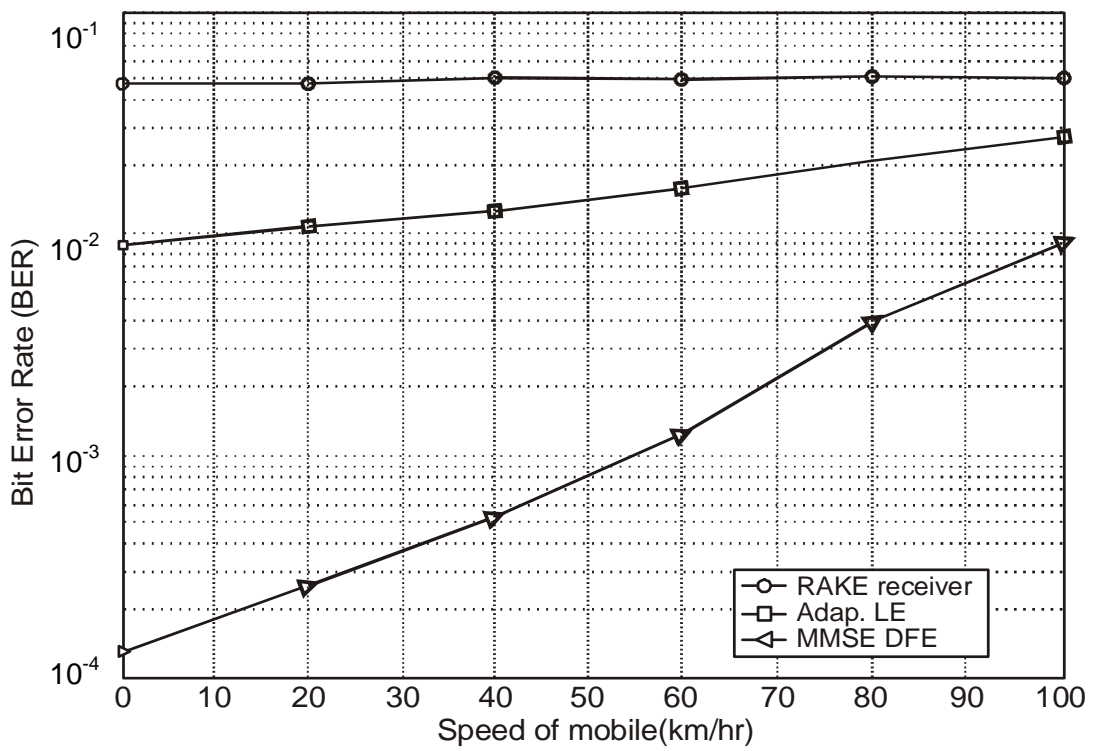

Figure 6. BER performance with respect to the speed of the mobile.

more efficiently compared to an adaptive LE and a RAKE receiver. It is also concluded that the structure of space-time coded MMSF DFE, when compared to other schemes of similar complexity, provides a reasonable balance between noise, ISI and MAI. Thus, this scheme works well when any of these three problems dominates. However, at very high interference levels, the use of transmit diversity does not improve the performance, unless the interference is suppressed by more sophisticated methods such as multi-user detection. As a whole, it is concluded that, in a wireless system, equalization, diversity, and STBC coding can be used together to boost the received signal quality and link performance. Although the system with only two transmit antennas is investigated here, it can be straightforwardly extended to a system with more transmit antennas. All these elegant results can be used for evaluating the system capacity, designing power control algorithms and appropriate channel coding techniques for WCDMA.

\section{References}

[1] S. Verdu, "Multiuser detection,” Cambridge University Press, New York, 1998.

[2] S. M. Razavizadeh, V. T. T. Vakili, and P. Azmi, “Comparison of several multiple antenna multiuser detectors in wireless CDMA system,” in Proceedings of the 5th IFIP TC6 Conference on Mobile and Wireless Networks (MWCN2003), 2003.

[3] Z. Zvonar, "Combined multiuser detection and diversity reception for wireless CDMA system,” IEEE Transactions on Vehicular Technology, Vol. 45, pp. 205-211, February 1996.

[4] S. M. Alamouti, "A simple transmit diversity technique for wireless communication,” IEEE Journal on Selected Areas in Communications, Vol.16, No. 8, pp. 1451-1458, October 1998.

[5] C. D. Frank and E. Visotsky, "Adaptive interference suppression for direct-sequence CDMA systems with long spreading codes," presented at the 36th Allerton Conference, 1998.

[6] A. Klein, "Data detection algorithms specially designed for the downlink of CDMA mobile radio system," in Proceedings of IEEE VTC-1997, pp. 203-207, 1997.

[7] F. Petre, M. Moonen, M. Engels, B. Gyselincky, and H. D. Man, "Pilot-aided adaptive chip equalizer receiver for interference suppression in DS-CDMA forward link," in Proceedings of IEEE VTC-Fall 2000, pp. 303-308, 2000.

[8] J. Wang and K. Yao, "Space-time coded wideband CDMA system," in Proceedings of VTC 2002, Vol. 1, pp. 260-264, Spring 2002.

[9] M. Abdulrahman, A. U. H. Sheikh, and D. D. Falconer, "Decision feedback equalization for CDMA in indoors wireless communication,” IEEE Journal on Selected Areas in Communications, Vol. 12, No. 4, pp. 698-706, May 1994.

[10] T. Udomsripariboon, C. Mingukwan, C. Benjangkaprasert, O. Sangaroon, and K. Janchitrapongvej, "Soft output decision feedback equalizer using variable step-size algorithm for turbo codes DS/CDMA systems,” in Proceedings of IEEE ISPACS, pp. 505-508, December 2005.

[11] S. Haykin and M. Moher, "Modern wireless communications,” Prentice-Hall, 2005.

[12] K. Takeda, K. Ishihara, and F. Adachi, "Downlink DSCDMA transmission with joint MMSE equalization and ICI cancellation,” Proceedings of IEEE VTC 2006-spring, Melbourne, Australia, Vol. 4, pp. 17621766, May 7-10, 2006. 